\title{
Oscillatory spin relaxation rates in quantum dots
}

\author{
C. F. Destefani and Sergio E. Ulloa \\ Department of Physics and Astronomy and Nanoscale and Quantum \\ Phenomena Institute, Ohio University, Athens, Ohio 45701-2979
}

(Dated: November 7, 2018)

\begin{abstract}
Phonon-induced spin relaxation rates in quantum dots are studied as function of in-plane and perpendicular magnetic fields, temperature and electric field, for different dot sizes. We consider Rashba and Dresselhaus spin-orbit mixing in different materials, and show how Zeeman sublevels can relax via piezoelectric and deformation potential coupling to acoustic phonons. We find that strong lateral and vertical confinements may induce minima in the rates at particular values of the magnetic field, where spin relaxation times can reach even seconds. We obtain good agreement with experimental findings in GaAs quantum dots.
\end{abstract}

PACS numbers: 71.70.Ej, 73.21.La, 72.25.Rb, 72.10.Di

Keywords: spin relaxation rate, acoustic phonon coupling, spin-orbit coupling, quantum dots

Since the proposal of a qubit based on the electron spin of quantum dots (QDs),, much work has been done to understand the processes that may cause their relaxation, since long coherence times are required. One of those processes is related to the phonon-induced spin-flip rates of Zeeman sublevels in QDs in magnetic fields, where the spin purity of the levels is broken by the spin-orbit (SO) interaction. A recent experiment ${ }^{2}$ has shown that spin relaxation time has a lower bound of $50 \mu \mathrm{s}$ at an in-plane field of $7.5 \mathrm{~T}$ in a GaAs QD defined in a 2DEG, while theoretical work ${ }^{3}$ has given $5 \mu$ s at the same QD parameters. In general, SO effects have been considered via perturbation theory ${ }^{4.5}$ although exact treatments have also been presented ${ }^{6.7 .8}$ The perturbative approach, which includes only a few states, has been called into question by the demonstration that a larger basis is needed in order to achieve convergence even for the lowest QD states when the QD vertical width is narrow ${ }^{7}$ where a complex interplay between different energy scales is present $\underline{\underline{9}}$ The influence of high SO coupling on the QD effective $g$-factor has been recently addressed. 10

Using exact diagonalization, our goal is to provide a bridge between the different approaches used so far, so that their range of validity can be well established in terms of materials (wide/narrow-gap), QD sizes, and directions of the magnetic field. For GaAs we show that the perturbative approach is indeed satisfactory for in-plane fields for any $Q D$ confinement, where good agreement is found with calculation ${ }^{3.6}$ and experiment, ${ }^{2}$ and where the piezoelectric potential is shown to dominate. If the field is perpendicular, however, we find that the perturbative approach ${ }^{4}$ indeed fails, giving relaxation rates orders of magnitude longer than our results if the $z$-confinement is strong $\stackrel{\underline{7}}{\underline{7}}$ For higher perpendicular fields, we show that minima are found in the rates depending on the QD features. For InSb the oscillatory rates are found for both in-plane and perpendicular fields and strong confinement. We find that the deformation potential dominates the rates, so that previous results $\underline{\underline{6}}^{\underline{\underline{m}}}$ under in-plane fields not including such potential yielded rates orders of magnitude smaller than ours. We show that the oscillatory behavior is caused by the nature of the $z$-confinement, as well as by the complex interplay between the physical scales playing a role in the system: Zeeman and SO energies, as well as QD lateral and vertical sizes.

The QD is defined by an in-plane parabolic confinement, $V(\rho)=m \omega_{0}^{2} \rho^{2} / 2$, where $m\left(\omega_{0}=E_{0} / \hbar\right)$ is the electronic effective mass (confinement frequency); the $\mathrm{QD}$ lateral length is $l_{0}=\sqrt{\hbar /\left(m \omega_{0}\right)}$. The vertical confinement $V(z)$ is strong enough so that only the state in the first quantum well subband is relevant, and its function is $\varphi_{z}(z)=\sqrt{2 / z_{0}} \sin \left(\pi z / z_{0}\right)$ if a hard wall is assumed, $z_{0}$ being the QD vertical well thickness. In a magnetic field $\mathbf{B}$, the unperturbed Hamiltonian, $H_{0}=\hbar^{2} \mathbf{k}^{2} / 2 m+V(\rho)+H_{Z}$, has the well-known Fock-Darwin (FD) solution, 11 where $H_{Z}=g_{0} \mu_{B} \mathbf{B} \cdot \sigma / 2$ is the Zeeman term. We include all $\mathrm{SO}$ terms in $2 \mathrm{D}$ zincblende QDs, namely Rashba 12 and Dresselhaus ${ }^{13}$ interactions. The former is due to surface inversion asymmetry (SIA) induced by the $2 \mathrm{D}$ confinement, while the latter is caused by the bulk inversion asymmetry (BIA) present in zincblende structures. The SIA Hamiltonian is $H_{S I A}=\alpha \sigma \cdot \nabla V(\rho, z) \times \mathbf{k}$, while the BIA is $H_{B I A}=$ $\gamma\left[\sigma_{x} k_{x}\left(k_{y}^{2}-k_{z}^{2}\right)+\sigma_{y} k_{y}\left(k_{z}^{2}-k_{x}^{2}\right)+\sigma_{z} k_{z}\left(k_{x}^{2}-k_{y}^{2}\right)\right]$, with coupling constants $\alpha$ and $\gamma$. The $z$-confinement yields the electric field $d V / d z$ in the SIA Hamiltonian as well as the momentum average $\left\langle k_{z}^{2}\right\rangle=\left(\pi / z_{0}\right)^{2}$ in the BIA terms. The full QD Hamiltonian is then $H=H_{0}+H_{S I A}+H_{B I A}$, which is diagonalized in a basis containing $110 \mathrm{FD}$ states. Details about the derivation of terms in $H$, as well as their selection rules for (anti)crossings in the QD energy spectrum, are found elsewhere $\underline{8.10}$

We calculate spin relaxation rates between the two lowest QD Zeeman sublevels caused by piezoelectric and deformation acoustic phonons via Fermi's Golden Rule: $\Gamma_{f i}=2 \pi / \hbar \sum_{j, \mathbf{Q}}\left|\gamma_{f i}(\mathbf{q})\right|^{2}\left|Z\left(q_{z}\right)\right|^{2}\left|M_{j}(\mathbf{Q})\right|^{2}\left(n_{Q}+\right.$ 1) $\delta\left(\Delta E+\hbar c_{j} Q\right)$, where the sum is over the emitted phonon modes $j(j=L A, T A 1, T A 2)$ with momentum $\mathbf{Q}=\left(\mathbf{q}, q_{z}\right)$. The term $Z\left(q_{z}\right)=\left\langle\varphi_{z}\left|e^{i q_{z} z}\right| \varphi_{z}\right\rangle$ $\left(\gamma_{f i}(\mathbf{q})=\left\langle f\left|e^{i \mathbf{q} \cdot \mathbf{r}}\right| i\right\rangle\right)$ is the form factor perpendicular (parallel) to the 2D-plane (position is $\mathbf{R}=(\mathbf{r}, z)$ ), while $n_{Q}$ is the phonon distribution with energy $\hbar c_{j} Q$; energies 
$\Delta E=\varepsilon_{f}-\varepsilon_{i}$ and states $|i\rangle,|f\rangle$ are obtained via diagonalization of the total $H$, so that the SO mixing is fully taken into account. The element $M_{j}(\mathbf{Q})=\Lambda_{j}(\mathbf{Q})+i \Xi_{j}(\mathbf{Q})$ includes both piezoelectric $\Lambda_{j}$ and deformation $\Xi_{j}$ potentials; in zincblende structures 14 and in cylindrical coordinates, they become $\Xi_{L A}(\mathbf{Q})=\Xi_{0} A_{L A} \sqrt{Q}$ (only $L A$ is present for $\left.\Xi_{j}\right), \Lambda_{L A}(\mathbf{Q})=3 \Lambda_{0} A_{L A} \sin (2 \theta) q^{2} q_{z} / 2 Q^{7 / 2}$, $\Lambda_{T A 1}(\mathbf{Q})=\Lambda_{0} A_{T A} \cos (2 \theta) q q_{z} / Q^{5 / 2}$, and $\Lambda_{T A 2}(\mathbf{Q})=$ $\Lambda_{0} A_{T A} \sin (2 \theta)\left(2 q_{z}^{2} / q^{2}-1\right) q^{3} / 2 Q^{7 / 2}$ (both $T A 1$ and $T A 2$ modes are compacted as a single $T A$ mode for $\left.\Lambda_{j}\right)$, where $A_{j}=\sqrt{\hbar\left(2 N_{0} V c_{j}\right)^{-1}}$ and $\Lambda_{0}=4 \pi e h_{14} / \kappa$. The bulk phonon constants are $\Xi_{0}$ and $e h_{14}, c_{j}$ are the sound velocities $\left(c_{T A 1}=c_{T A 2}=c_{T A} \neq c_{L A}\right), \kappa$ is the dielectric constant, and $N_{0}$ is the electron density. The triple space integration $\left(\left[r, \phi_{r}, z\right]\right)$ yields an analytical solution, $\underline{\underline{7}}$ and we were able to do two $\left(\left[\phi_{q}, q_{z}\right]\right)$ out of the momentum integration, leaving a numerical integral only in $q$. For a later use, the only $z_{0}$-dependence in this remaining integral in $\Gamma_{f i}$ reads $F_{j}\left(z_{0}\right)=\left(d_{j} z_{0}-\left(d_{j} z_{0}\right)^{3} / \pi^{2}\right)^{-2} \sin ^{2}\left(d_{j} z_{0}\right)$, where $d_{j}=$ $\sqrt{\left(\Delta E / \hbar c_{j}\right)^{2}-q^{2}} / 2 ; q$ runs from 0 to $\Delta E / \hbar c_{j}$, while $F_{j}\left(z_{0}\right)$ is multiplied by polynomials and exponentials in $q$ in the total $\Gamma_{f i}$. No approximation is needed in our derivation of $\Gamma_{f i}$, so that the $3 \mathrm{D}$ nature of the phonon is taken into account and the full form factor $e^{i \mathbf{Q} \cdot \mathbf{R}}$ is used.

Panel $A$ of Fig. 1 shows the phonon-induced spin-flip rates as a function of in-plane field, $B_{\|}$, for different values of $E_{0}$ (solid lines) for GaAs QDs ${ }^{15}$ Larger $E_{0}$ (smaller QDs) present smaller spin-flip rates, i.e., longer relaxation times. This happens because as $E_{0}$ increases, the orbital levels become more separated and then the SO coupling becomes relatively less important. A strong dependence of the rates with $B_{\|}$and $E_{0}$ is clear. For example, at $B_{\|}=2 \mathrm{~T}$, one gets $2.5 \times 10^{2} \mathrm{~s}^{-1}$ at $E_{0}=0.7$ $\mathrm{meV}$ and $2.5 \times 10^{-1} \mathrm{~s}^{-1}$ at $E_{0}=5.0 \mathrm{meV}$; at $B_{\|}=15 \mathrm{~T}$ the rate goes from $5.2 \times 10^{6} \mathrm{~s}^{-1}$ to $6.9 \times 10^{4} \mathrm{~s}^{-1}$ for those same values of $E_{0}$. Finite temperature (dashed-dotted line for $T=12 \mathrm{~K}$ ) enhances the rates by about one order of magnitude due to the enhancement of the phonon population. Larger wells (dotted line for $z_{0}=100 \AA$ ) have rates about one order of magnitude smaller because the BIA term $\left(\left\langle k_{z}^{2}\right\rangle \approx 1 / z_{0}^{2}\right)$ decreases. The same $T$ and $z_{0}$ behavior is found for any $E_{0}$. Larger electric fields (dashed line for $d V / d z=-38 \mathrm{meV} / \AA$ ) have little effect on the rates for large $E_{0}$, but at smaller $E_{0}$ the rates decrease by one order of magnitude; this means that by increasing the SIA term so that it equals the BIA coupling, the rates can decrease even though the spin mixing gets stronger. For GaAs QDs under in-plane fields, we find that the TA piezoelectric coupling dominates at low fields $\left(\lesssim 14 \mathrm{~T}\right.$ for $E_{0}=5 \mathrm{meV}$ ), while at high fields the deformation potential takes over (symbols in Fig. 11A).

Panels $B$ and $C$ of Fig. 1 respectively show the spin expectation value $\left\langle\sigma_{z}\right\rangle$ of the Zeeman sublevels and their energy splitting $\Delta E$. Smaller $E_{0}$ values show higher SOinduced spin mixing, so that $\left\langle\sigma_{z}\right\rangle$ deviates from the pure $+1(-1)$ value for the ground (first excited) state, and
FIG. 1: $(A)$. Zeeman sublevel spin-flip rates for GaAs QDs ${ }^{15}$ under in-plane magnetic field for different QD confinements (solid lines). Dashed-dotted, dotted, and dashed lines respectively show influence of higher temperature, well width, and electric field on the $3.5 \mathrm{meV}$ QD. Symbols show isolated contributions from the distinct phonon mechanisms for the 5.0 meV QD; TA piezoelectric dominates at low fields. (B). Spin average values of the Zeeman sublevels after diagonalization of $H$. Squares (circles) refer to ground (first excited) state. $(C)$. Energy splitting of Zeeman sublevels after diagonalization of $H$. Dashed-dotted lines show results without SO coupling. Color scheme in panels $B$ and $C$ follow from panel $A$.

FIG. 2: Same as Fig. 11 but for GaAs QDs under perpendicular magnetic field. Vertical lines in panel $B$ show the field where a sublevel crossing occurs and the normal spin character of the states is restored. Arrows in panel $C$ indicate where suppression of SO coupling is induced by the magnetic field, which is at the origin of the respective minima in panel $A$.

$\Delta E$ deviates from the pure $g_{0} \mu_{B} B$ value of a QD without SO coupling. Notice at the $3.5 \mathrm{meV}$ QD how a larger well (stronger field) decreases (increases) the SO-induced spin mixing by reducing (enhancing) the importance of the BIA (SIA) coupling. For comparison, the experimental lower bound for the spin-flip time is $50 \mu \mathrm{s}$ at $B_{\|}=7.5$ $\mathrm{T}$ for a $1.1 \mathrm{meV} \mathrm{QD}, \stackrel{2}{,}$ while from panel $A$ we find $5 \mu \mathrm{s}$. As discussed above, this time is increased by one order of magnitude in a larger $z$-well, which appears to be a better match for the experimental conditions. A $5 \mu$ s value has also been found from a perturbative formulation, ${ }^{3}$ and our results also agree with a different model for the vertical confinement $\stackrel{6}{\underline{6}}$ One can then affirm that the perturbative approach ${ }^{4}$ is indeed enough for $\mathrm{GaAs}$ in inplane fields at any QD sizes, and that the form of the $z$-confinement has little effect on the rates.

In Fig. 2 we do the same analysis for GaAs QDs 15 in a perpendicular field, $B_{\perp}$. The most striking feature is the appearance of minima in the rates shown in panel $A$. The $E_{0}$-dependent minima $\left(12 \leq B_{\perp} \leq 16 \mathrm{~T}\right)$ are due to the vanishing $\Delta E$, as shown in panel $C$. At such values of $B_{\perp}$, a level crossing of Zeeman sublevels results in a sudden spin-flip, as verified by the vertical lines of panel $B$ for the 0.7 and $3.5 \mathrm{meV}$ QDs. To understand the minima at low fields $\left(6 \leq B_{\perp} \leq 8 \mathrm{~T}\right)$ we have to be mindful of the sine argument in $F_{j}\left(z_{0}\right)$, which may induce a minimum in the rate at a particular value of the field, due to the interplay of energy and length scales in the problem. 9 This behavior does not produce minima for in-plane field on GaAs QDs (Fig. 11). We find that those three low-field minima in Fig. 22 $A$ occur at $B_{\perp}$ values where a magnetic field-induced cancellation of the SO influence is produced on the respective $\Delta E$ values, as indicated by the arrows in panel $C$ : below (above) such $E_{0}$-dependent values of $B_{\perp}$ - where $\Delta E$ recovers its Zeeman value of $g_{0} \mu_{B} B-$ the SO coupling increases (decreases) the Zeeman splitting as compared to the QD without SO. 
FIG. 3: InSb QDs ${ }^{17}$ under in-plane magnetic field. The confinements are such that the same QD size-range used for GaAs is covered. In panel $A$, the $15.0(20.0) \mathrm{meV}$ QD is chosen for the study of different QD parameters (distinct phonon mechanisms); dashed arrows show that both LA modes have the same number of rate minima and happen at the same fields.

Notice that the two smallest confinements do not show the low-field rate minima in Fig. 2 $A$. This is due to the lowest sublevels acquiring the same spin (between 3 and $7 \mathrm{~T}$ at $E_{0}=0.7 \mathrm{meV}$ in panel $B$ ), so that the rate decreases monotonically at that field-range. These features have strong influence on QD effective $g$-factors ${ }^{10}$ Like in the in-plane field problem, higher temperatures $(T=12$ $\mathrm{K}$ ) enhance the rates by one order of magnitude, and the dominating phonon mechanism (symbols) is the TA piezoelectric coupling, but now at any field. To confirm the intricate interplay between all QD energy scales, a larger well $\left(z_{0}=100 \AA\right)$ or a stronger field $(d V / d z=-38$ $\mathrm{meV} / \AA)$ removes the rate minima, as shown in panel $A$ for the $3.5 \mathrm{meV} \mathrm{QD}$. Our results agree with available calculations ${ }^{7}$ at small fields $\left(B_{\perp} \leq 1 \mathrm{~T}\right)$, so that we can confirm that the perturbative approach is not adequate when dealing with SO effects in QDs in a perpendicular field, even if the material is GaAs, if the vertical confinement is strong enough. ${ }^{16}$ From Figs. 10and 2 the rates at small fields $(\lesssim 2 \mathrm{~T})$ have the same behavior under $B_{\|}$ and $B_{\perp}$, namely, the smaller $E_{0}$ the larger the rate; however, they are much larger under $B_{\perp}$. The rate maximum (at $2.5 \mathrm{~T}$ for the $1.1 \mathrm{meV} \mathrm{QD}$ ) is due to an anticrossing involving the first and second QD excited states 10

Figure 3 shows results for InSb QDs17 in in-plane fields. In contrast to GaAs (Fig. (1), minima in the rates are visible under $B_{\|}$for the highest $E_{0}$ values. At small fields $(\lesssim 2 \mathrm{~T})$, a monotonic rate drop is again observed as $E_{0}$ increases. As shown for the $20 \mathrm{meV} \mathrm{QD}$, the LA deformation potential is the dominant phonon mechanism for $I n S b$ at any field (and $E_{0}$ ); this seems to be the case for any narrow-gap material 18 It is worth mentioning that, contrary to GaAs in Fig. 24 where a unique lowfield minimum is present for a given $E_{0}$, the number of rate minima in InSb increases with $E_{0}$. Also, notice that both LA modes have the same number of minima at the same $B_{\|}$values (dashed arrows in panel $A$ ), while a larger number of minima in the TA mode occurs at different fields; this happens since $c_{L A}>c_{T A}$, so that the sine argument in $F_{j}\left(z_{0}\right)$ is smaller in the $L A$ mode. Panel $B$ shows that Zeeman sublevels have normal spin character, where ground (excited) state is predominantly spin-up (spin-down) at low fields. Finite temperature $(T=12$ K) has no appreciable effect on the rates, a reflection of the much larger energy scales here. The energy scale interplay results at higher field $(d V / d z=-2 \mathrm{meV} / \AA)$ in slightly smaller (larger) $\Delta E$ (rates), and rate minimum shifts to higher fields. On the other hand, a larger well $\left(z_{0}=100 \AA\right)$ produces a higher $\Delta E$, so that the spin-flip rate is suppressed by orders of magnitude and its num-

FIG. 4: Same as Fig. 3 but for InSb QDs under perpendicular magnetic field. The only minimum of the $3.0 \mathrm{meV} \mathrm{QD}$ around $1 \mathrm{~T}$ in panel $A$ indicates a sublevel crossing, as shown by the vertical line in panel $B$ and the vanishing of $\Delta E$ in panel $C$. The color scheme is the same in all panels and figures.

ber of minima increases; notice in panel $B$ that the two lowest levels acquire the same spin at $\simeq 8 \mathrm{~T}$ for such $z_{0}$, so that the oscillatory rate is well defined until that field. Previous calculations ${ }^{6}$ in InSb did not include the deformation potential, and yielded unrealistically low rates.

For InSb QDs in perpendicular fields, we see from panel $B$ in Fig. 4 that all spin-flip rates in panel $A$ are meaningful only for $B_{\perp}<8 \mathrm{~T}$, a field where both Zeeman sublevels acquire the same spin. This happens earlier for small $E_{0}$ as shown by the $3.0 \mathrm{meV}$ QD, whose rates are well defined only for $B_{\perp}<2 \mathrm{~T}$; the vertical line around $1 \mathrm{~T}$ in panel $B$ for this $E_{0}$ indicates a spin-flip, accompanied by the vanishing $\Delta E$ in panel $C$, the rate minimum in panel $A$, and a sign change of the QD $g$-factor ${ }^{10}$ Larger values of $E_{0}$ present normal spin behavior. Spin-flip rates in panel $A$ do not show the monotonic behavior seen for small fields in Fig. [3 Like in the $B_{\|}$case, a finite temperature $(T=12 \mathrm{~K})$ does not show visible influence on the rates under $B_{\perp}$, and the deformation potential dominates at any field. A higher electric field $(d V / d z=-2 \mathrm{meV} / \AA)$ slightly increases the rate; a larger well $\left(z_{0}=100 \AA\right)$ introduces an oscillatory rate orders of magnitude smaller. We emphasize that the perturbative approach finds no use in InSb QDs because of the inherent higher SO coupling. It is worth mention that oscillatory rates have also been found in GaAs QDs under $B_{\perp}$ by considering momentum relaxation from an excited orbital level to the ground state,$\frac{19}{9}$ as well as in coupled GaAs QDs ${ }^{20}$

Even though both QD materials show oscillatory spinflip rates - with $B_{\perp}$ for GaAs and with both $B_{\|}$and $B_{\perp}$ for InSb - their origin is slightly different. Minima come from the nature of the $z$-confinement, and the field where they occur depend on the lateral size $l_{0}$. The SO coupling mixes spins and alters splitting of sublevels in distinct ways according to field-direction and QD material, so that spin relaxation can be induced by piezoelectric (wide-gap) and deformation (narrow-gap) phonons. Our calculations reveal the rich interplay between all relevant energy scales in QDs. The external field opens channels (at the rate minima) where long spin relaxation times $(\simeq 1 \mathrm{~s})$ may be reached, so that the spin coherence required for quantum computing could be improved.

We thank support from NSF-IMC grant 0336431, CMSS at OU, and the $21^{\text {st }}$ Century Indiana Fund. 
1 D. Loss and D. P. DiVincenzo, Phys. Rev. A 57, 120 (1998).

${ }^{2}$ R. Hanson, B. Witkamp, L. M. K. Vandersypen, L. H. W. van Beveren, J. M. Elzerman, and L. P. Kouwenhoven, Phys. Rev. Lett. 91, 196802 (2003).

${ }^{3}$ V. N. Golovach, A. Khaetskii, and D. Loss, Phys. Rev. Lett. 93, 016601 (2004).

4 A. V. Khaestkii, Y. V. Nazarov, Phys. Rev. B 64, 125316 (2001); L. M. Woods, T. L. Reinecke, and Y. LyandaGeller, Phys. Rev. B 66, 161318(R) (2002).

5 D. V. Bulaev and D. Loss, cond-mat/0409614

${ }^{6}$ R. de Sousa and S. Das Sarma, Phys. Rev. B 68, 155330 (2003).

7 J. L. Cheng, M. W. Wu, and C. Lü, Phys. Rev. B 69, 115318 (2004).

8 C. F. Destefani, S. E. Ulloa, and G. E. Marques, Phys. Rev. B 69, 125302 (2004); C. F. Destefani, S. E. Ulloa, and G. E. Marques, Phys. Rev. B 70, 205315 (2004).

9 M. Valín-Rodríguez, Phys. Rev. B 70, 033306 (2004).

10 C. F. Destefani and S. E. Ulloa, cond-mat/0411071

11 L. Jacak, A. Wojs, and P. Hawrylak, Quantum Dots (Springer, Berlin, 1998).

12 Y. A. Bychkov and E. I. Rashba, J. Phys. C 17, 6039 (1984).
13 G. Dresselhaus, Phys. Rev. 100, 580 (1955).

14 V. F. Gantmakher and Y. B. Levinson, Carrier Scattering in Metals and Semiconductors (North-Holland, Amsterdam, 1987).

15 In GaAs: $m=0.067 \mathrm{~m}_{0}, g_{0}=-0.44, \kappa=12.4, \alpha=$ $4.4 \AA^{2}, \gamma=26 \mathrm{eV} \AA^{3}, \Xi_{0}=7 \mathrm{eV}, e h_{14}=0.140 \mathrm{eV} / \AA$, $N_{0}=5.32 \times 10^{-27} \mathrm{Kg} / \AA^{3}, c_{L A}=4.73 \times 10^{13} \AA / \mathrm{s}$, and $c_{T A}=3.35 \times 10^{13} \AA / \mathrm{s} ;$ also, $z_{0}=40 \AA, d V / d z=-0.5$ $\mathrm{meV} / \AA$, and $T=0 \mathrm{~K}$ if no other numbers are specified.

${ }^{16}\left\langle k_{z}^{2}\right\rangle=\left(\pi / z_{0}\right)^{2}$ for an infinite well. If a Gaussian well is considered, one has $\left\langle k_{z}^{2}\right\rangle=1 / z_{0}^{2}$, which yields a BIA term a factor of 10 smaller than used in this work.

17 In InSb: $m=0.014 \mathrm{~m}_{0}, g_{0}=-51, \kappa=16.5, \alpha=500 \AA^{2}$, $\gamma=160 \mathrm{eV}^{3}, \Xi_{0}=7 \mathrm{eV}, e h_{14}=0.061 \mathrm{eV} / \AA, N_{0}=5.77 \times$ $10^{-27} \mathrm{Kg} / \AA^{3}, c_{L A}=3.40 \times 10^{13} \AA / \mathrm{s}$, and $c_{T A}=2.29 \times 10^{13}$ $\AA / \mathrm{s} ;$ also, $z_{0}=40 \AA, d V / d z=-0.5 \mathrm{meV} / \AA$, and $T=0 \mathrm{~K}$ if no other numbers are specified.

18 A. M. Alcalde, Qu Fanyao, and G. E. Marques, Phys. E 20, 228 (2004).

19 U. Bockelmann, Phys. Rev. B 50, 17271 (1994).

20 A.Bertoni, M. Rontani, G. Goldoni, F. Troiani, and E. Molinari, Appl. Phys. Lett. 85, 4729 (2004). 

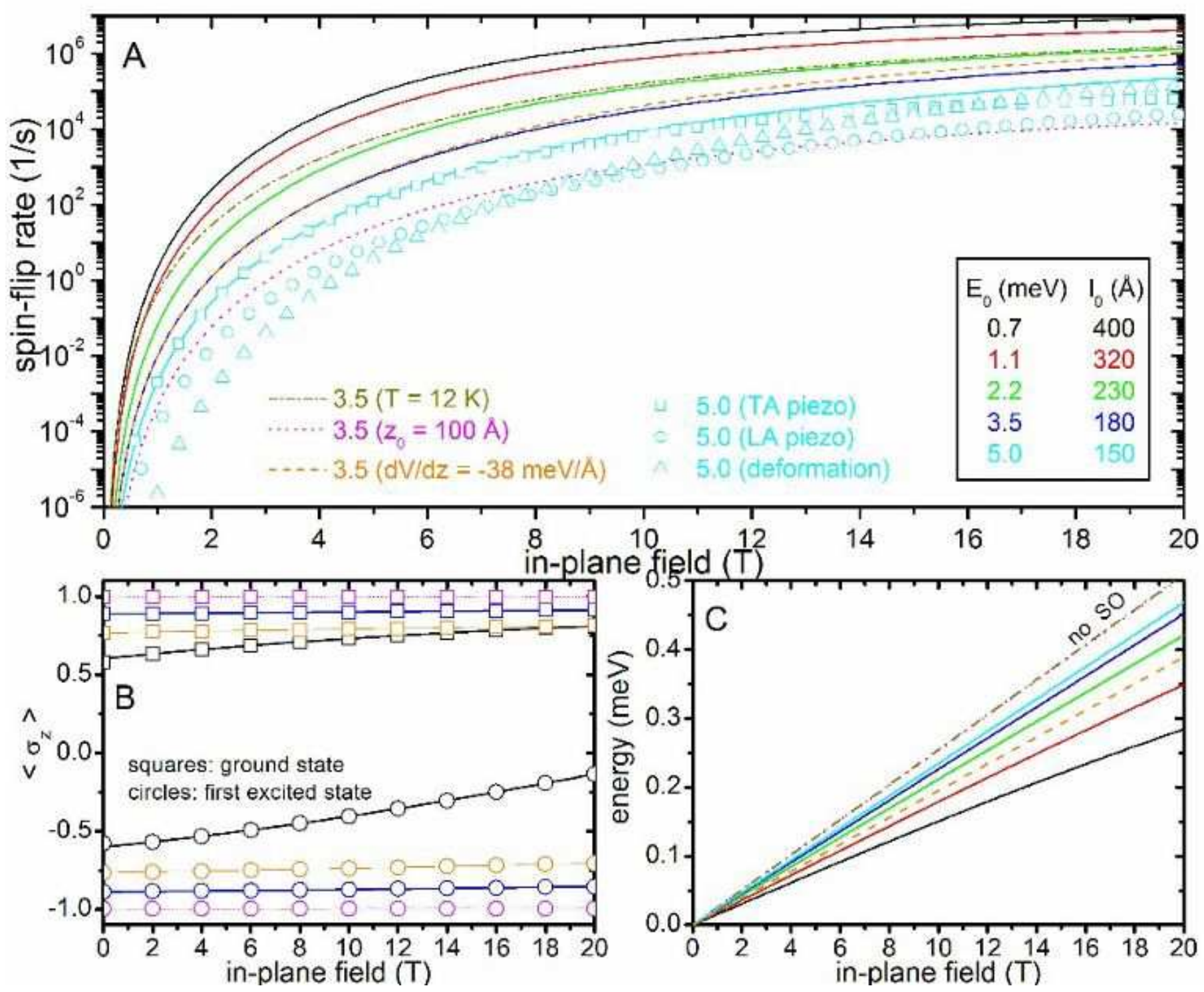

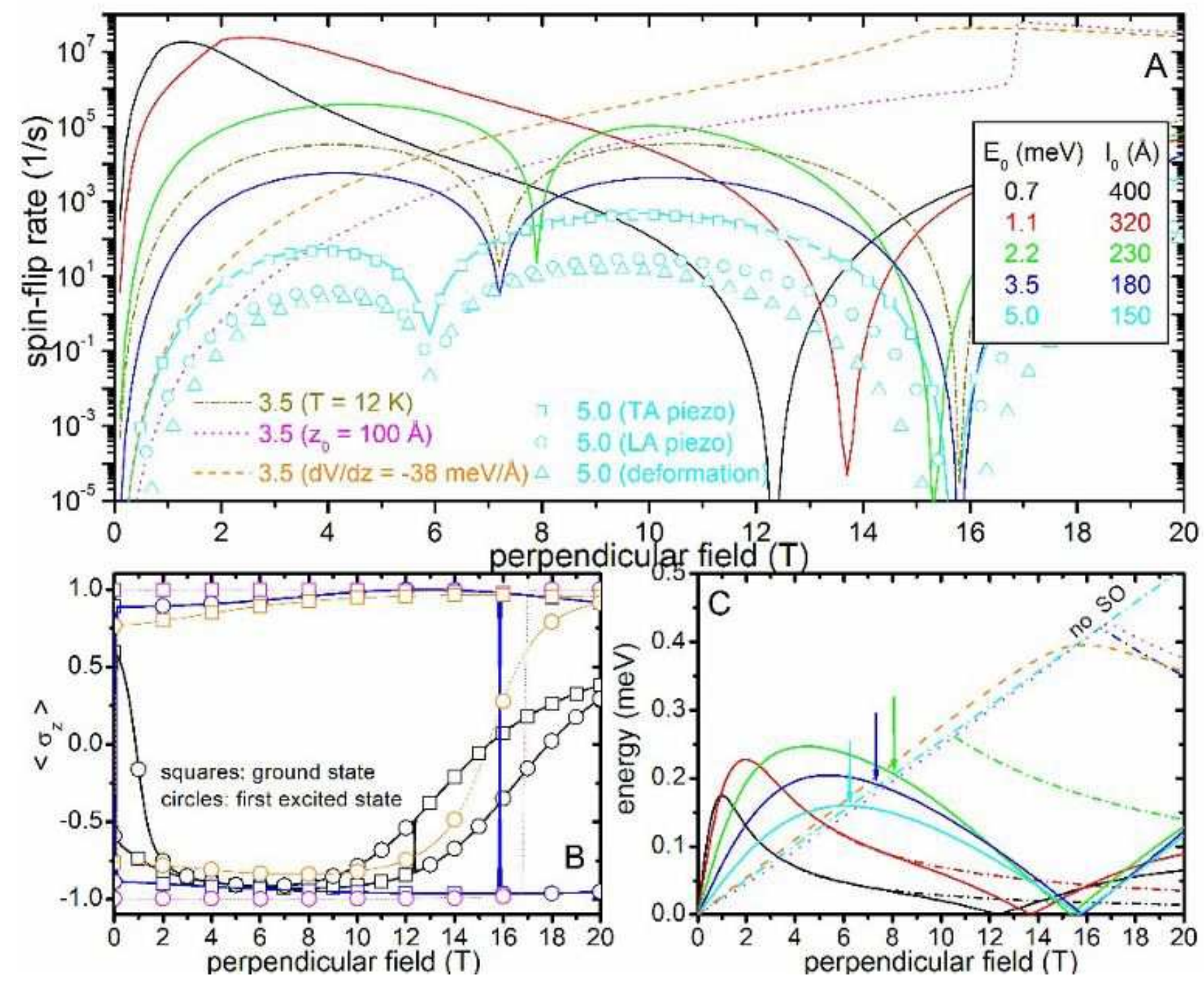

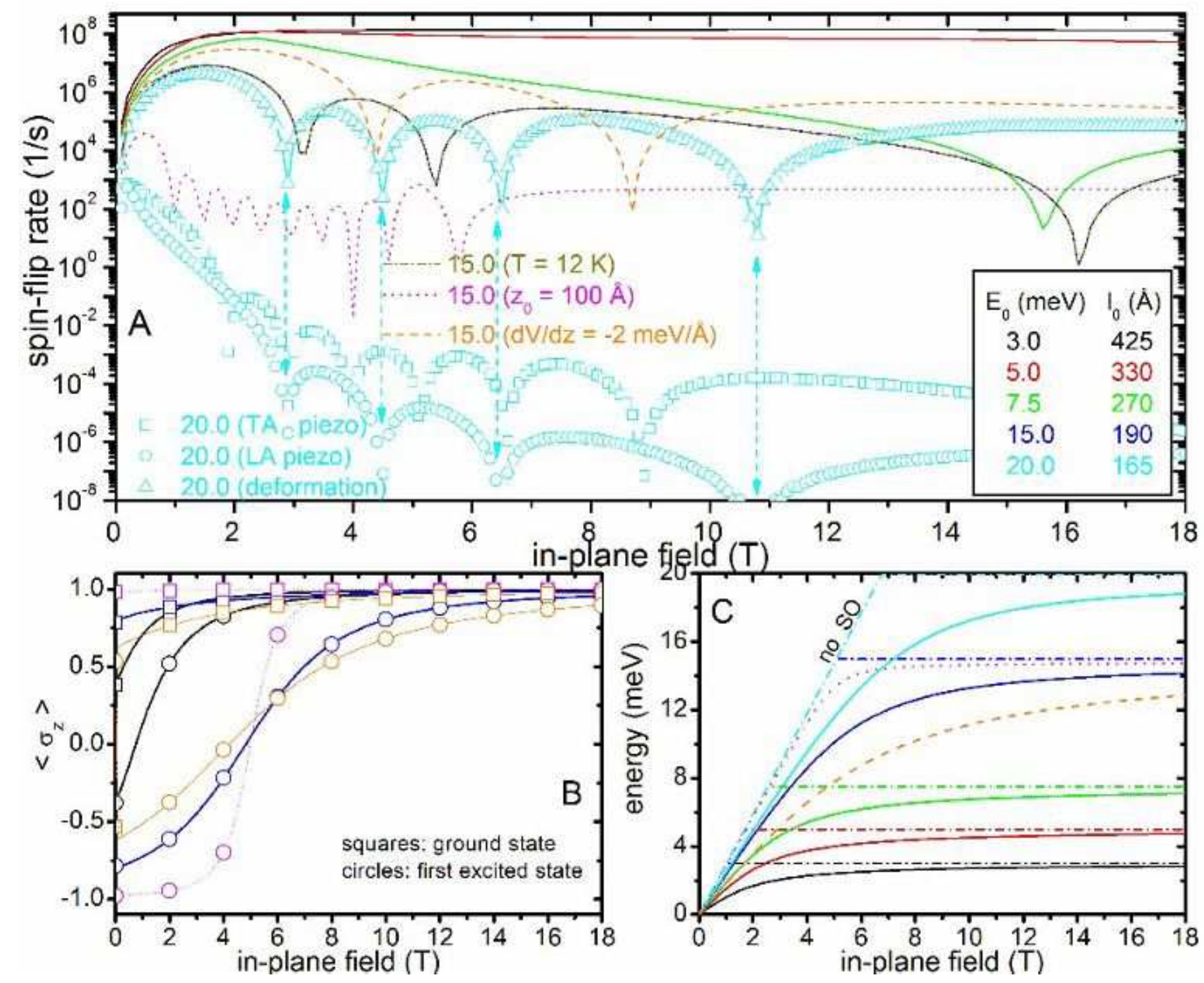

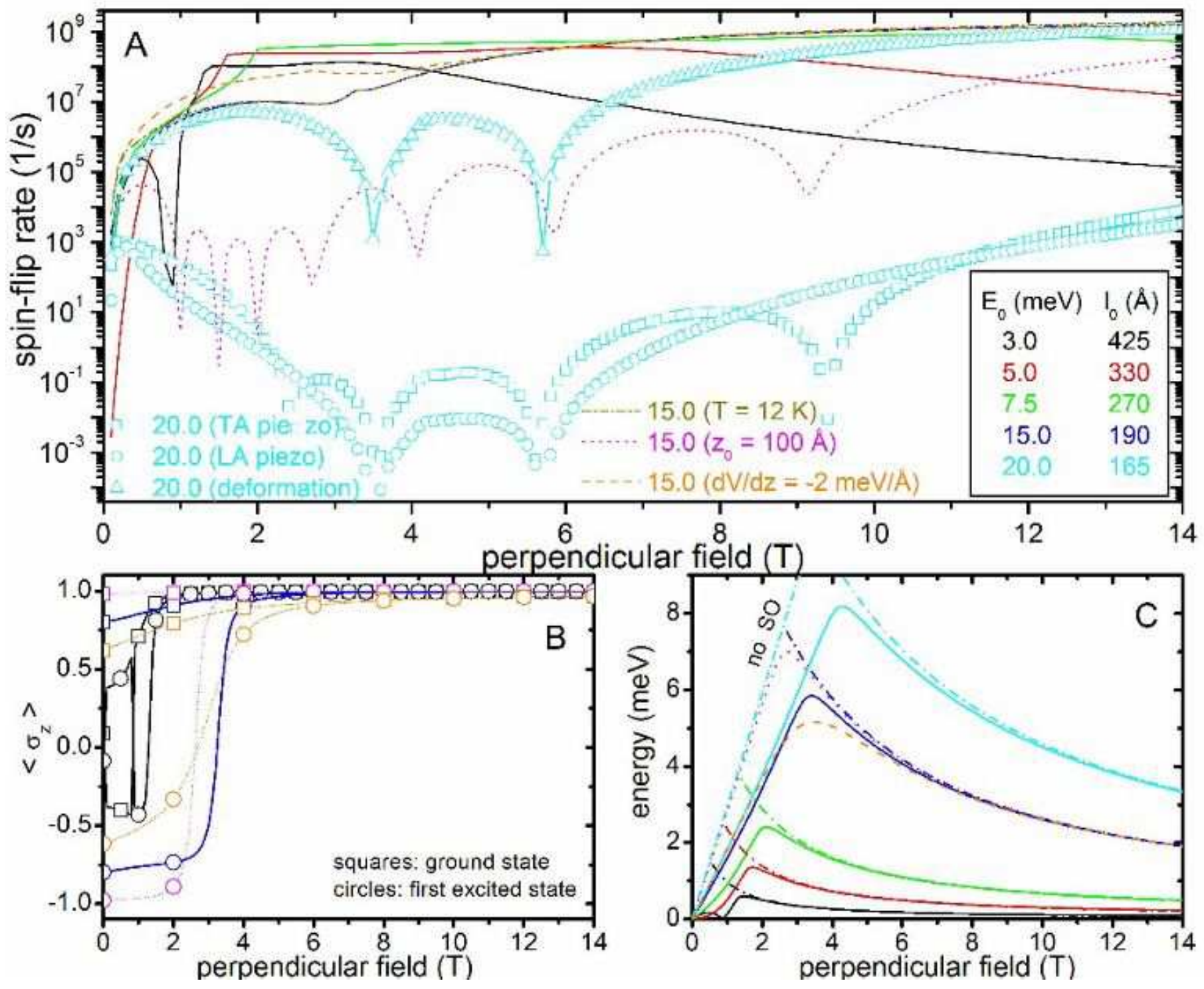